\section{BMJ Open} Ophthalmology

\title{
Evaluation of the safety and effectiveness of oral propranolol in patients with von Hippel-Lindau disease and retinal hemangioblastomas: phase III clinical trial
}

\author{
Beatriz González-Rodríguez, ${ }^{1}$ Karina Villar Gómez de las Heras, ${ }^{\circledR 2,3}$ \\ Daniel T Aguirre, ${ }^{4}$ Luis Rodríguez-Padial, ${ }^{5}$ Virginia Albiñana, ${ }^{6,7}$ \\ Lucía Recio-Poveda, ${ }^{6}$ Angel M Cuesta, ${ }^{6,7}$ Luisa M ${ }^{a}$ Botella, ${ }^{6,7}$ \\ Rosa María Jiménez-Escribano ${ }^{1}$
}

\begin{abstract}
To cite: González-Rodríguez B, Villar Gómez de las Heras K, Aguirre DT, et al. Evaluation of the safety and effectiveness of oral propranolol in patients with von Hippel-Lindau disease and retinal hemangioblastomas: phase III clinical trial. BMJ Open Ophthalmology 2019;4:e000203. doi:10.1136/ bmjophth-2018-000203
\end{abstract}

- Additional material is published online only. To view please visit the journal online (http://dx.doi.org/10.1136/ bmjophth-2018-000203).

Two posters about this work were presented at the 9th European Conference on Rare Diseases \& Orphan Products (ECRD 2018 Vienna) and at the 13th International VHL Medical/Research Symposium (MD Anderson Cancer Center, Houston, Texas, USA, 2018).

Received 13 August 2018 Revised 21 February 2019 Accepted 19 March 2019

Check for updates

(c) Author(s) (or their employer(s)) 2019. Re-use permitted under CC BY-NC. No commercial re-use. See rights and permissions. Published by BMJ.

For numbered affiliations see end of article.

Correspondence to Dr Karina Villar Gómez de las Heras; kvillar@jccm.es

\section{ABSTRACT}

Background von Hippel-Lindau disease (VHL) is a multisystem cancer syndrome caused by mutations in the $V H L$ gene. Retinal hemangioblastoma is one of the most common tumours, and when it appears near the optic nerve, its treatment is challenging and risky. To date, no treatment has proven effective in changing the course of the disease. This study was designed to evaluate the safety and effectiveness of propranolol in controlling these tumours.

Methods Seven patients were included. All patients took a daily dose of $120 \mathrm{mg}$ of propranolol for 1 year. Clinical variables were assessed at baseline, and at 1, 3, 6, 9 and 12 months. The primary endpoint of the study was the number and size of retinal hemangioblastomas. On every visit, retinal outcomes and blood biomarkers (such as vascular endothelial growth factor (VEGF) and miR210) were analysed.

Results Number and size of retinal hemangioblastomas remained stable in all patients. All of them had initially increased levels of VEGF and miR210. There was a gradual reabsorption of retinal exudation in two patients, correlating with a progressive decrease of both biomarkers. The only adverse effect reported was hypotension in one patient.

Conclusions Propranolol could be used to treat retinal hemangioblastomas in VHL patients, although more studies are needed to determine the ideal dose and long-term effect. VEGF and miR210 should be explored as biomarkers of disease activity. As far as we know, these are the first biomarkers proposed to monitor the VHL disease activity. Trial registration number 2014-003671-30

\section{BACKGROUND}

Genetics and molecular basis

Von Hippel-Lindau (VHL) disease is a rare autosomal dominant disease with an incidence of 1 in 36000 births. ${ }^{1}$ It is caused by germline mutations in the tumour suppressor gene VHL (3p25-26), which lead to the

\section{Key messages}

What is already known about this subject?

Standard treatments for retinal hemangioblastoma involve laser photocoagulation and cryotherapy, among others.

- There is no safe treatment for juxtapapillary tumours.

- Up to now, no treatment has proven effective in changing the course of the disease.

\section{What are the new findings?}

- All retinal tumours in all patients remained stable in number and size during the treatment with propranolol P.0.

- There was a gradual reabsorption of exudates in two patients.

- This event correlated with a progressive decrease of VEGF and miRNA210 levels in all patients, until reaching normal levels similar to those found in the general population.

How might these results change the focus of research or clinical practice?

- Propranolol could be a pharmacological treatment for retinal hemangioblastomas, particularly useful when exudates are present.

VEGF and miRNA210 could be used as potential biomarkers to monitor disease activity in VHL and the response to pharmacological treatments.

Although further studies are necessary in order to confirm its clinical usefulness, these results should be considered by researchers investigating other diseases where exudates are involved.

development of different tumours throughout life: retinal and central nervous system (CNS) hemangioblastomas, renal cancer, pheochromocytomas/paragangliomas, endolymphatic sac tumours, pancreatic cystadenomas and neuroendocrine tumours, cystadenomas in the epididymis and broad ligament. ${ }^{12}$ 
VHL patients are heterozygous at birth, and only cells that undergo a second-hit somatic mutation of the wildtype allele (loss of heterozygosity) ${ }^{3}$ will develop tumours. ${ }^{4}$ This loss of heterozygosity will cause a subsequent loss of function of the protein complex/ubiquitin kinase of which pVHL is a key element. This results in accumulation of hypoxia-inducible factors (HIFs) within the cytoplasm and their secondary translocation to the cell nucleus, where they initiate the transcription of a number of genes involved in cell proliferation, angiogenesis, vascular tone and erythropoiesis, among other processes. ${ }^{5}$ The increased expression of these factors is associated with the development of highly vascular tumours. ${ }^{6}$

\section{Ophthalmic characteristics}

Retinal hemangioblastomas are a well-recognised finding in VHL. They are among the most frequent $(49 \%$ to $85 \%)^{7}$ and earliest presenting tumours in the disease, so the ophthalmologist is necessarily involved in the follow-up and care of these patients. ${ }^{27}$

Most hemangioblastomas are symptomatic at presentation, ${ }^{8}$ one-third of patients have multiple tumours and up to half the patients have bilateral involvement. ${ }^{7}$ The most frequent location is the peripheral retina, although they can be found juxtapapillary. ${ }^{9}$

Early tumours are small red-round lesions located between an arteriole and a venule. Well-established tumours are seen as a round orange-red mass, located in the temporal periphery with dilatation, and tortuosity of the supplying artery and draining vein extending from the optic disc. Secondary effects of retinal hemangioblastoma are predominantly exudative $(25 \%)$ causing leakage with macular exudate, or tractional (9\%), causing retinal detachment. Macular pucker is observed in $9 \%$ of the eyes. Other complications include vitreous haemorrhage, secondary glaucoma, phthisis bulbi and loss of the eye. ${ }^{7}$

The diagnosis can be made by screening of patients at risk or in symptomatic patients with macular exudate or retinal detachment. Funduscopy, fluorescein angiography and optical coherence tomography (OCT) are useful for the diagnosis.

Without treatment, and sometimes regardless of the standard treatments, these lesions continue to grow and affect the visual function. ${ }^{9}$

Depending on the size and the location, there are different treatment options. The visual outcome is greatly dependent on the size, location and number of tumours and the presence of complications such as exudative or tractional retinal detachment. ${ }^{7}$ Small peripheral lesions are usually treated with laser photocoagulation, while in larger ones-especially those with exudative retinal detachment-cryotherapy is applied. Photodynamic therapy has been used with uneven results, such as anti-vascular endothelial growth factor (VEGF) drugs (bevacizumab, ranibizumab), which do not provide longterm cessation of tumour growth. ${ }^{10}$ Other treatments include brachytherapy. Potential complications, such as non-absorbing vitreous haemorrhage, epiretinal fibrosis and tractional retinal detachment, can be treated by vitreoretinal surgery and, if necessary, endolaser photocoagulation. ${ }^{11}$

Treatment of juxtapapillary and optic nerve hemangioblastomas is particularly difficult and risky. As tumours may remain quiescent for many years and due to the high risk of iatrogenic visual loss with standard treatments, ${ }^{11}$ in asymptomatic juxtapapillary hemangioblastomas without exudation, only monitoring is recommended.

To date, no pharmacological treatment has proven effective in changing the course of the disease. ${ }^{12}$

\section{In vitro assays}

Propranolol is a well-known non-selective $\beta$-blocker agent marketed for more than 50 years, used for cardiac and neurological diseases. ${ }^{12}{ }^{13}$ It has proven effective in the treatment of infantile hemangioma, and nowadays it is considered its first-line treatment. It has also been tested in some malignant tumours. The results of different studies support the role of $\beta$-adrenergic signalling pathways in the regulation of breast tumour progression, ${ }^{14-17}$ and-as it inhibits cell proliferation on in vitro cell cultures-it has been proposed a role on antiangiogenesis and antitumour effects. ${ }^{5}{ }^{12}{ }^{15-17}$ A recent publication describes its off-label use as an adjuvant treatment in melanoma, where it seems to protect patients from recurrence, what could lead to a decrease in patients' mortality. ${ }^{18}$

In a previous work, our group developed primary cell cultures from surgically resected CNS hemangioblastomas and performed in vitro assays with propranolol. ${ }^{5}$ We demonstrated that propranolol decreased the expression of HIF target genes in hemangioblastoma cells and affected their viability, probably acting as an antiangiogenic agent inhibiting VEGF, and as a proapoptotic drug. Based on these previous outcomes, we wanted to evaluate the safety and effectiveness of propranolol for the treatment of retinal hemangioblastomas in VHL patients, to determine if retinal hemangioblastomas decrease in size or remain stable compared with baseline, after the completion of the treatment period.

\section{METHODS}

We designed an open clinical trial, with only one arm of treatment, to evaluate the safety and effectiveness of propranolol in VHL patients with retinal hemangioblastomas.

The association of patients Alianza VHL informed about and invited their members to participate in the study. The participants should meet one of the following two criteria:

1. Papillary or juxtapapillary hemangioblastomas, non-eligible for standard treatment (laser photocoagulation or cryotherapy) due to the high risk of iatrogenic visual loss.

2. Peripheral retinal hemangioblastomas for which patients had rejected standard treatments. 
Seven VHL patients from different regions of Spain were included. All of them had a positive genetic diagnosis of VHL. Four patients had previous visual impairment as a result of exudation due to active tumours. Some of them had undergone previous treatments, like laser photocoagulation or cryotherapy.

The pharmaceutical form and strength used was propranolol $40 \mathrm{mg}$, film-coated tablets, 1 every 8 hours up to a total dosage of $120 \mathrm{mg} /$ day, achieved in $7-10$ days. An experienced cardiologist in handling propranolol established the dosage regimen. The patients did not receive other treatments during the study.

The primary endpoint of the study was the number and size of all the retinal hemangioblastomas. The secondary endpoints were visual acuity, biomicroscopy, intraocular pressure, funduscopy, OCT, exudation and exudative retinal detachment.

The follow-up visits were scheduled at the Ophthalmology Department, Virgen de la Salud Hospital (Toledo), at baseline and at months 1, 3, 6, 9 and 12 of treatment. At each visit, an ophthalmologist performed the measurement of the endpoints and took fundus photographs. Regardless of the clinical trial, these patients continued the controls by their regular ophthalmologists.

The secondary objective of the study was to determine if the selected dosage of propranolol was effective in controlling the growth of CNS hemangioblastomas. The neurosurgeon evaluated the MRI with contrast, obtained from the brain and spinal cord, before and after the study completion.

The exploratory objectives were the detection and quantification of plasma biomarkers VEGF and miR210 along the clinical trial, and the detection of HIF-controlled targets in the peripheral blood leukocytes. These biomarkers were evaluated to check a possible relationship between their levels and the disease activity. For this aim, a blood sample was extracted from every patient in each visit and sent to Dr Botella's lab (CIB-CSIC).
Additional biomarkers such as BAX, Cas-9, Epo, Sox-2 and Oct- 4 were also tested as a control of the propranolol effect.

\section{RESULTS}

Table 1 shows the summary of patients' characteristics, and online supplementary table 1 shows the main data obtained during the follow-up visits. Number and size of all retinal tumours remained stable in all patients. There was an unexpected finding, a progressive reabsorption of retinal exudates in the two patients who had them initially (patients 1 and 3, figures 1 and 2, respectively). The clearest reabsorption occurred in patient 1 , being almost complete after 6 months of treatment.

Patient 6 had a previous macular pucker with severe visual impairment. She had a retinal scheduled surgery and was admitted to the trial in an attempt to avoid it. Finally, she withdrew after 6 months to undergo surgery.

The only adverse effect reported was hypotension suffered by patient 4 , so there was a slower dose increase to achieve the target dosage of $120 \mathrm{mg}$.

No significant changes were found in the growth pattern of CNS hemangioblastomas (supplementary table 2). Only two patients remained stable regarding the oedema and the cystic or nodular component of their hemangioblastomas. None of them required neurosurgery along the clinical trial.

As previously reported, ${ }^{12}$ along the trial, VEGF protein levels significantly decreased from the first month of treatment, reaching normal levels (concentration $<50$ $\mathrm{pg} / \mathrm{mL}$ ) in all cases after 3 months of treatment. There was an increase in the Bax gene expression, a proapoptotic gene, and a reduction in Epo, Sox-2 and Oct-4, all genes involved in angiogenesis and stemness. miR210 decreased at the beginning of the treatment.

After the end of the trial, patients 1, 2, 5 and 7 decided to continue taking propranolol off-label. Some time later, no new exudates, new tumours or growth of the preexistent ones were reported in these patients (see

Table 1 Summary of patients' characteristics

\begin{tabular}{|c|c|c|c|c|c|c|c|c|}
\hline \multirow[b]{3}{*}{ Patient } & \multirow[b]{3}{*}{ Age and gender } & \multicolumn{4}{|l|}{ First visit } & \multirow[b]{3}{*}{ Adverse effects } & \multirow{2}{*}{\multicolumn{2}{|c|}{ Last visit }} \\
\hline & & \multicolumn{2}{|l|}{ Left eye } & \multicolumn{2}{|l|}{ Right eye } & & & \\
\hline & & Tumours & Exudation & Tumours & Exudation & & Tumours* & Exudation \\
\hline 1 & $36 \mathrm{M}$ & One periph. & - & One periph. & Yes & - & No changes & No \\
\hline 2 & $33 \mathrm{~F}$ & One juxtap. & - & - & - & - & No changes & - \\
\hline 3 & $50 \mathrm{~F}$ & One juxtap. & Yes & - & - & - & No changes & Less exudation \\
\hline 4 & $20 \mathrm{M}$ & One juxtap. & - & - & - & Hypotension & No changes & - \\
\hline 5 & $15 \mathrm{M}$ & - & - & One periph. & - & - & No changes & - \\
\hline 6 & $38 \mathrm{~F}$ & One juxtap. & - & - & - & - & Withdrawn† & - \\
\hline 7 & $22 \mathrm{M}$ & Two periph. & - & Two periph. & & - & No changes & - \\
\hline
\end{tabular}

The table exclusively includes active tumours in the initial or final visit, which was the principal objective of the treatment.

*'No changes' indicates no new tumours or growth of previous ones.

†Withdrawn from the clinical trial due to a scheduled surgery.

Juxtap, Juxtapapillary; Periph, peripheral. 


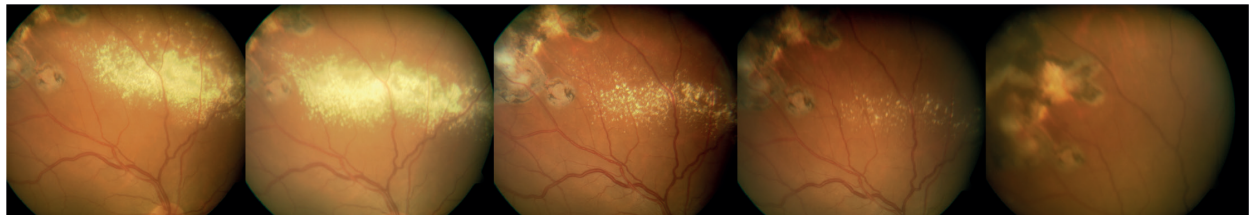

Figure 1 Patient 1. Reabsorption of exudation. The first image corresponds to the baseline visit and the last one after 1 year of treatment. This patient had the highest baseline levels of VEGF in peripheral blood and experienced the highest reduction during the follow-up, decreasing to those found in general population. VEGF, vascular endothelial growth factor.

supplementary table 1). Patient 5 finally discontinued the medication and 3 months later had to undergo a serial of different treatments due to an increase in tumoural activity, with a subsequent loss of vision.

\section{DISCUSSION}

We present the results of a clinical trial designed to test an old drug-propranolol-to treat a common manifestation of a rare disease-VHL-for which there is no pharmacological treatment. Four of our patients had juxtapapillary tumours, and therefore a high risk of iatrogenic visual loss if treated. Others had refused more standard treatments because of the tumour progression despite previous treatment.

Even though we may think that our findings could be due to tumour quiescence or just the natural course of VHL disease, spontaneous regression of retinal exudates is an unusual event in the absence of a specific treatment.

On the other hand, the reabsorption of retinal exudation correlated with the highest reductions of VEGF protein levels measured in peripheral blood in patient 1 , so we may attribute these findings to the propranolol effect. This fact could be explained by the role proposed for propranolol in angiogenesis, ${ }^{15}$ since it decreases VEGF levels, produces vasoconstriction and leads to cellular apoptosis. ${ }^{19} 20$ All these effects may be contributing to stabilisation or regression of hemangioblastoma tumour cells. miR210 had a parallel evolution to VEGF. We could consider plasma levels of VEGF and miR210 as potential biomarkers for disease activity in VHL, and they may also be good for monitoring the response to a possible treatment, although further studies are necessary in order to confirm its clinical usefulness.

As complementary information, to give strength to our data, we asked the patients for a copy of their clinical histories, so we could draw their clinical evolutions. Only three patients could retrieve this interesting information, represented in figure 3. The beginning of the clinical trial is marked by a black line.

Propranolol is a well-known and cheap drug with few adverse effects, which could be used in rare diseases like VHL, where in some cases, despite multiple treatments for the tumour and their complications, patients suffer from vision loss. These results provide us with the basis to explore higher doses of propranolol-up to $3 \mathrm{mg} / \mathrm{kg}$ body weight/day, adjusting dose by body weight-and even consider the use of a more selective $\beta$-blocker that could be more effective. It may be interesting as well to explore the effect of intravitreal injection of propranolol in clinical trials, as the case report recently published ${ }^{21}$ and based on the previous work by Nourinia et al, to determine the safe dose of intravitreal propranolol. ${ }^{22}$ Using intravitreal administration, the drug would achieve more easily the retina.

Although a recent publication supports our hypothesis that propranolol reduces hemangioblastoma growth, ${ }^{23}$ more studies are needed and with more patients to confirm these results, preferably with fewer previous invasive treatments (such as laser photocoagulation).

\section{CONCLUSIONS}

Our outcomes suggest that propranolol could be useful for the treatment of retinal hemangioblastomas in VHL patients, especially when retinal exudates are present, but more studies are needed to confirm its possible therapeutic value in this group of patients.

Plasma levels of VEGF and miR210 should be explored as biomarkers of the disease activity. If confirmed, they will be the first biomarkers to monitor the VHL disease.

It would also be interesting to explore the effect of propranolol on other diseases where exudation and/or high VEGF levels are present.

The results of this clinical trial led to the first international orphan drug designation to treat von

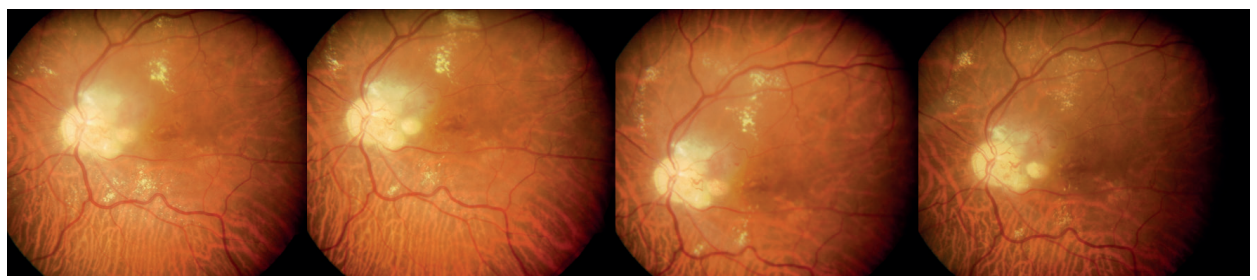

Figure 2 Patient 3. Partial reabsorption of retinal exudation. The images show the evolution in a patient with a juxtapapillary hemangioblastoma, before treatment and after 1 year of treatment with oral propranolol (last image, right). 


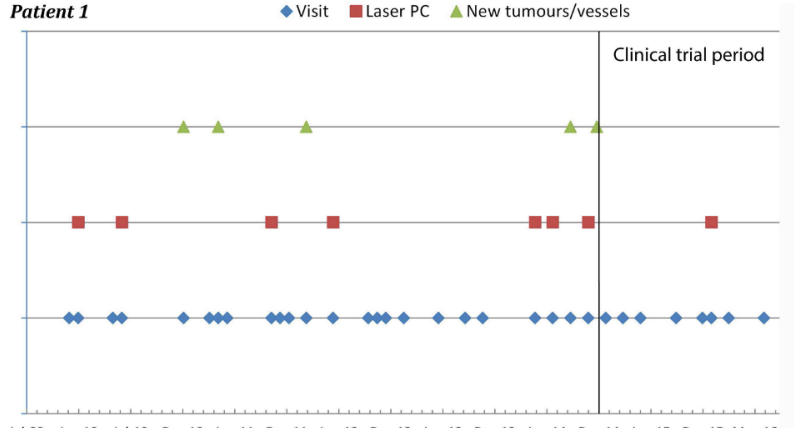

Jul-09 Jan-10 Jul-10 Dec-10 Jun-11 Dec-11 Jun-12 Dec-12 Jun-13 Dec-13 Jun-14 Dec-14 Jun-15 Dec-15 May-16 Patient 2

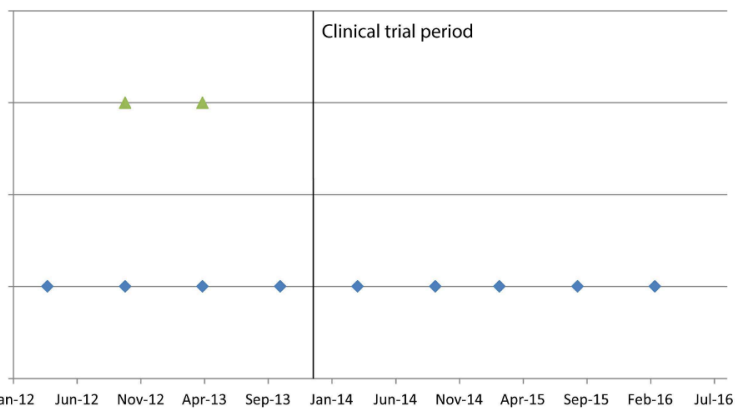

$\begin{array}{llllllllll} & \text { Jan-12 Jun-12 Nov-12 Apr-13 } & \text { Sep-13 Jan-14 Jun-14 } & \text { Nov-14 } & \text { Apr-15 } & \text { Sep-15 } & \text { Feb-16 } & \text { Jul-16 }\end{array}$ Patient 5

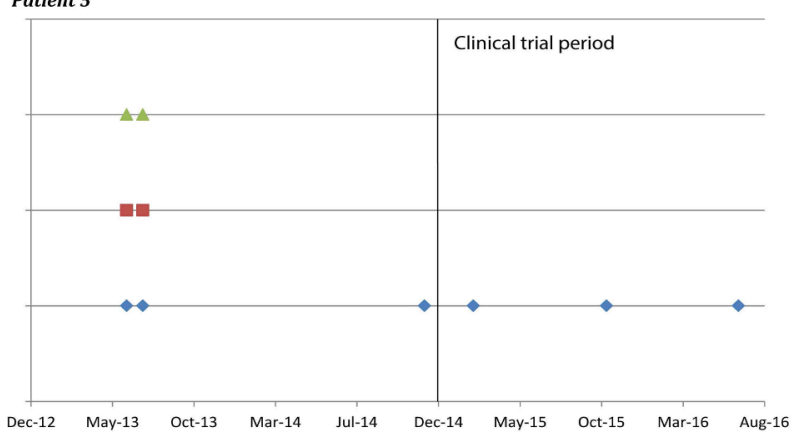

Figure 3 Patients 1, 2 and 5. Clinical evolution before and during the clinical trial. Based on the notes written by their regular ophthalmologists in the clinical histories. The black lines mark the beginning of the trial.

Hippel-Lindau disease (EU/3/17/1841), granted by the European Medicines Agency (EMA) for propranolol, January 2017.

\section{Author affiliations}

${ }^{1}$ Ophthalmology, Retina Service, Complejo Hospitalario de Toledo, Servicio de Salud de Castilla-La Mancha, Toledo, Spain

${ }^{2}$ DG Asistencia Sanitaria, Servicio de Salud de Castilla-La Mancha, Toledo, Spain

${ }^{3}$ Alianza VHL, Alianza de Familias de von Hippel-Lindau, Toledo, Spain

${ }^{4}$ Neurosurgery, Familial Neuro-Oncology Unit, Hospital Universitario Fundación Jiménez Díaz, Madrid, Spain

${ }^{5}$ Cardiology, Complejo Hospitalario de Toledo, Servicio de Salud de Castilla-La Mancha, Toledo, Spain

${ }^{6}$ Centro de Investigaciones Biológicas—CIB, Centro Superior de Investigaciones Científicas (CSIC), Madrid, Spain

${ }^{7}$ Centro de Investigación Biomédica en Red de Enfermedades Raras (CIBERER), Madrid, Spain

Acknowledgements We are grateful to the patients who participated in our research and to the Health Service of Castilla-La Mancha (SESCAM) for supporting the performance of the clinical trial at the Virgen de la Salud Hospital (Toledo). Special thanks to Susi Martínez (president of Alianza VHL), for all her work with patients and researchers; and Mercedes Mota Pérez (SESCAM), who provided advice in the preparation of the clinical trial protocol.

Contributors KV and RMJ planned the study. KV wrote the clinical trial protocol and carried out all the authorisation processes and contact with patients. LR participated in the selection of patients to assure they could take propranolol. He performed the cardiological evaluations and follow-up, from a cardiologic point of view. RMJ participated in the evaluation of patients to determine their eligibility according the inclusion criteria. She directed and coordinated the clinical visits, follow-up and complementary diagnostic tests. RMJ and BG performed the ophthalmological evaluations, some of them after the end of the clinical trial. DTA performed the neurological evaluations. LMB directed and coordinated the in vitro experiments, the design strategies and the experimental approach. VA and AMC carried out the experimental work, assisted by Lucia R. BG, RMJ and KV analysed the clinical results. BG wrote the first draft of the document and $\mathrm{KV}$ reviewed it and added content. KV, BG and AMC worked in the first review of the document. KV and $B G$ worked in improving the final version of the manuscript. All authors read and approved the final manuscript.

Funding This clinical trial was supported by grants from Alianza VHL Spain, Fundación Iberdrola, Bodegas Protos and Fundación Port Aventura. Alianza VHL informed the families about the clinical trial for the recruitment of patients, performed the administrative procedures for authorisation and monitoring and financed the travel expenses of the patients for the visits.

Competing interests None declared.

Patient consent for publication Not required.

Ethics approval The protocol study and informed consent form were reviewed and approved by the Ethical Committee of the Complejo Hospitalario de Toledo. The study was classified by the Spanish Agency of Medicines and Medical Devices (AEMPS) as a phase III clinical trial, authorised, and registered in EU Clinical Trials Register (EMA).

Provenance and peer review Not commissioned; externally peer reviewed.

Open access This is an open access article distributed in accordance with the Creative Commons Attribution Non Commercial (CC BY-NC 4.0) license, which permits others to distribute, remix, adapt, build upon this work non-commercially, and license their derivative works on different terms, provided the original work is properly cited, appropriate credit is given, any changes made indicated, and the use is non-commercial. See: http://creativecommons.org/licenses/by-nc/4.0/.

\section{REFERENCES}

1. Lonser RR, Glenn GM, Walther M, et al. Von Hippel-Lindau disease. The Lancet 2003;361:2059-67.

2. Wong WT, Agrón E, Coleman HR, et al. Clinical characterization of retinal capillary hemangioblastomas in a large population of patients with von Hippel-Lindau disease. Ophthalmology 2008;115:181-8.

3. Gläsker Set al. Reconsideration of biallelic inactivation of the VHL tumour suppressor gene in hemangioblastomas of the central nervous system. J Neurol Neurosurg Psychiatry 2001;70:644-8.

4. Knudson AG. Cancer genetics. Am J Med Genet 2002;111:96-102.

5. Albiñana V, Villar Gómez de Las Heras K, Serrano-Heras G, et al. Propranolol reduces viability and induces apoptosis in hemangioblastoma cells from von Hippel-Lindau patients. Orphanet J Rare Dis 2015;10:118

6. Findeis-Hosey JJ, McMahon KQ, Findeis SK, et al. Von HippelLindau disease. J Pediatr Genet 2016;5:116-23.

7. Singh AD, Shields CL, Shields JA. Von Hippel-Lindau disease. Surv Ophthalmol 2001;46:117-42.

8. Chittiboina P, Lonser RR. Von Hippel-Lindau disease. Handb Clin Neurol 2015;132:139-56.

9. Chew EY. Ocular manifestations of von Hippel-Lindau disease: clinical and genetic investigations. Trans Am Ophthalmol Soc 2005; 103:495-511.

10. Wong WT, Liang KJ, Hammel K, et al. Intravitreal ranibizumab therapy for retinal capillary hemangioblastoma related to von HippelLindau disease. Ophthalmology 2008;115:1957-64.

11. Kanski JJ, Bowling B. Kanski's Clinical Ophthalmology. A Systematic Approach. Elsevier Health Sciences, 2011.

12. Albiñana V, Escribano RMJ, Soler I, et al. Repurposing propranolol as a drug for the treatment of retinal haemangioblastomas in von Hippel-Lindau disease. Orphanet J Rare Dis 2017:12:122.

13. García-Sevilla J, Barturen F. Fármacos que modifican la actividad simpática. In: Farmacología Humana. 3rd ed. Barcelona: Masson, 1997: 275-6.

14. Powe DG, Voss MJ, Zänker KS, et al. Beta-blocker drug therapy reduces secondary cancer formation in breast cancer and improves cancer specific survival. Oncotarget 2010;1:628-38. 
15. Pasquier E, Ciccolini J, Carre M, et al. Propranolol potentiates the anti-angiogenic effects and anti-tumor efficacy of chemotherapy agents: implication in breast cancer treatment. Oncotarget 2011;2:797-809.

16. Barron $\mathrm{TI}$, Connolly RM, Sharp L, et al. Beta blockers and breast cancer mortality: a population- based study. $J$ Clin Oncol 2011;29:2635-44

17. Ganz PA, Habel LA, Weltzien EK, et al. Examining the influence of beta blockers and ACE inhibitors on the risk for breast cancer recurrence: results from the LACE cohort. Breast Cancer Res Treat 2011;129:549-56.

18. De Giorgi V, Grazzini M, Benemei S, et al. Propranolol for off-label treatment of patients with melanoma: results from a cohort study. JAMA Oncol 2018;4:e172908.
19. Akbar S, Alsharidah MS. Are beta blockers new potential anticancer agents? Asian Pac J Cancer Prev 2014;15:9567-74.

20. Storch $\mathrm{CH}$, Hoeger PH. Propranolol for infantile haemangiomas: insights into the molecular mechanisms of action. $\mathrm{Br} J$ Dermatol 2010;163:269-74.

21. Karimi $\mathrm{S}$, Nikkhah $\mathrm{H}$, Ahmadieh $\mathrm{H}$, et al. Intravitreal injection of propranolol for the treatment of retinal capillary hemangioma in a case of von Hippel-Lindau. Retin Cases Br Rep 2018;0.

22. Nourinia R, Rezaei Kanavi M, Kaharkaboudi A, et al. Ocular safety of intravitreal propranolol and its efficacy in attenuation of choroidal neovascularization. Invest. Ophthalmol. Vis. Sci. 2015;56:8228-35.

23. Shepard MJ, Bugarini A, Edwards NA, et al. Repurposing propranolol as an antitumor agent in von Hippel-Lindau disease. $J$ Neurosurg 2018;(Oct 26):1-9. 